\title{
Deep subwavelength Fabry-Perot-like resonances in a sandwiched reflection grating
}

\author{
C. P. Huang, ${ }^{1,2, *}$ X. G. Yin, ${ }^{3}$ Y. Zhang, ${ }^{2}$ S. B. Wang, ${ }^{1}$ Y. Y. Zhu, ${ }^{4}$ H. Liu, ${ }^{4}$ and C. T. Chan ${ }^{1, \dagger}$ \\ ${ }^{1}$ Department of Physics, The Hong Kong University of Science and Technology, Clear Water Bay, Kowloon, Hong Kong, China \\ ${ }^{2}$ Department of Applied Physics, Nanjing University of Technology, Nanjing 210009, China \\ ${ }^{3}$ State Key Laboratory of Millimeter Waves, City University of Hong Kong, Tat Chee Avenue, Kowloon, Hong Kong, China \\ ${ }^{4}$ National Laboratory of Solid State Microstructures, Nanjing University, Nanjing 210093, China \\ (Received 29 January 2012; revised manuscript received 5 May 2012; published 6 June 2012)
}

\begin{abstract}
A Fabry-Perot (FP) resonance cavity can be made to be subwavelength by manipulating the reflection phase at the boundaries. Here, we employ the open-ended slit cavities within a simple sandwiched reflection grating and show that a deep subwavelength FP-like resonance in the slits can be realized. An FP cavity with the length and width of tens of nanometers, which is two orders of magnitude smaller than the resonance wavelength, has been suggested. The effect is very robust and can operate from infrared to microwave frequencies.
\end{abstract}

DOI: $10.1103 /$ PhysRevB.85.235410

PACS number(s): 42.25.Bs, 42.79.Dj, 73.20.Mf

\section{INTRODUCTION}

By utilizing strong resonances, light can be confined to subwavelength objects. For example, a metal nanoparticle can confine and guide the flow of visible light using localized surface-plasmon resonances. ${ }^{1,2}$ Such subwavelength confinement of light is crucial for realizing compact photonic devices and various field enhancement effects. Recently, plasmonic metamaterials consisting of subwavelength components have attracted considerable attention. ${ }^{3}$ These artificial structures can be used to realize exotic physical properties such as optical magnetism, ${ }^{4}$ negative optical refraction, ${ }^{5}$ superlensing, ${ }^{6}$ and/or zero or high refractive index. ${ }^{7,8}$ Transformation optics facilitates the design of graded and anisotropic metamaterials, which guide light to flow in curved trajectories and can be used to realize invisibility. ${ }^{9,10}$

A Fabry-Perot (FP) resonance can also be used to confine light. The FP resonance is typically supported by a close-ended cavity with two reflecting boundaries, and, as such, the cavity length is comparable to or larger than the wavelength. This makes the device bulky and hence not suitable for applications that require compact or small form-factor components. It was suggested that subwavelength FP resonators may be created with reflectors that induce phase shifts different from 0 or $\pi{ }^{11}$ In recent years, by manipulating the reflection phase with the metamaterial reflectors, the subwavelength cavities have been demonstrated in the microwave band. ${ }^{12-14}$ Similar effects could be used to realize perfect metamaterial absorbers, employing a periodic particle array placed closely to a metal surface. ${ }^{15-17}$

As is well known, single slits or slit arrays cut into a metal film can also behave like the common FP cavities ${ }^{18-21}$ as long as the slit length is large enough. Such an FP resonance may give rise to enhanced optical or acoustic transmission (EOT/EAT) ${ }^{20,21}$ In this paper we suggest that by manipulating the effective reflection phase, the metallic slits within a simple sandwiched reflection grating act as "deep subwavelength" FP cavities, where the cavity size can be two orders of magnitude smaller than the resonance wavelength. Compared with the previous designs, our FP cavity is open-ended without the specially designed reflectors at the openings. Thus the cavity can be constructed easily with the nanosized length and width and can operate at high frequencies. The effect may be utilized to drive mechanical oscillations, detect tiny motion with ultrahigh sensitivity, and has a whole host of other potential applications at deep subwavelength scale.

\section{RESULTS AND DISCUSSIONS}

The schematic view of structure under study is shown in Fig. 1. Here, an air-filled metallic slit grating and a planar metal film, with the metal thickness much larger than the skin depth, are separated by a thin air spacer (the qualitative conclusion does not depend on the filling dielectric medium in the spacer). The structure is characterized by the lattice period $d$, slit width $a$, slit length $h$, and spacer thickness $t$. The structure is illuminated by a TM-polarized light, where the permittivity of incident side is $\varepsilon_{d}$, the incident angle is $\varphi$, and the magnetic field acts along the $z$ axis. Similar structures have been investigated previously in the regime $h \ll t,{ }^{22-25}$ where the spacer region below the metal stripes is dominant and the horizontal FP-like resonances in the spacer can be established. But here we focus on the opposite regimes, i.e., $h \gg t$ and $\lambda \gg h$. We will show that the metallic slits may act as deep subwavelength FP cavities that can trap the incident light efficiently.

To reveal the optical properties of the structure, we simulated the reflection spectrum at normal incidence using the commercial software CST Microwave Studios. Here, the incident side was assumed to be glass with $\varepsilon_{d}=2.25$; the metal (gold) was modeled with a Drude dispersion $\varepsilon_{m}=1-\omega_{p}^{2} / \omega(\omega+i \gamma)$, where $\omega_{p}=1.37 \times 10^{16} \mathrm{rad} / \mathrm{s}$ and $\gamma=5 \times 10^{13} \mathrm{~Hz}$. Without loss of generality, the structure parameters were set as $d=1500 \mathrm{~nm}, a=50 \mathrm{~nm}$, and $t=20 \mathrm{~nm}$. We tested slit lengths $h$ of $80 \mathrm{~nm}, 300 \mathrm{~nm}$, and $500 \mathrm{~nm}$. The simulation results are shown in Fig. 2(a) by the symbols, where a series of reflection dips can be seen (we will mainly focus on the long wavelength resonances). For example, a dominant dip is observed at $\lambda=6400 \mathrm{~nm}$ with a reflection efficiency of $3 \%$ for $h=80 \mathrm{~nm}$. Since there is no transmission and the high-order reflecting modes are all evanescent (when $\lambda>2250 \mathrm{~nm}$ ), the low reflection means $97 \%$ of incident light can be collected and absorbed by a metal slab with slit openings that cover only $3 \%$ of the area. An increase of slit length $h$ causes the reflection 


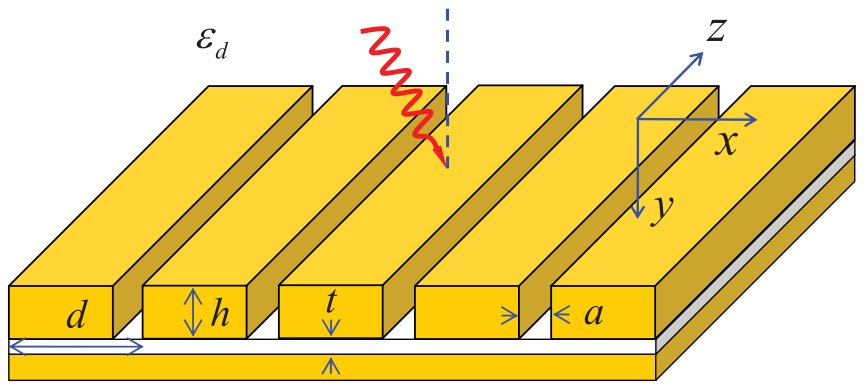

FIG. 1. (Color online) Schematic view of the plasmonic slab structure. A thin air spacer separates a planar metal film and a metallic slit grating. The incident light is TM-polarized with the magnetic field along the $z$ axis.

dip to redshift significantly. For example, a change of $h$ from $80 \mathrm{~nm}$ to $500 \mathrm{~nm}$ results in a spectral redshift of $2380 \mathrm{~nm}$. This effect cannot be understood simply as "horizontal" FP-like resonances, which are determined by the metal stripe width and air-spacer thickness.
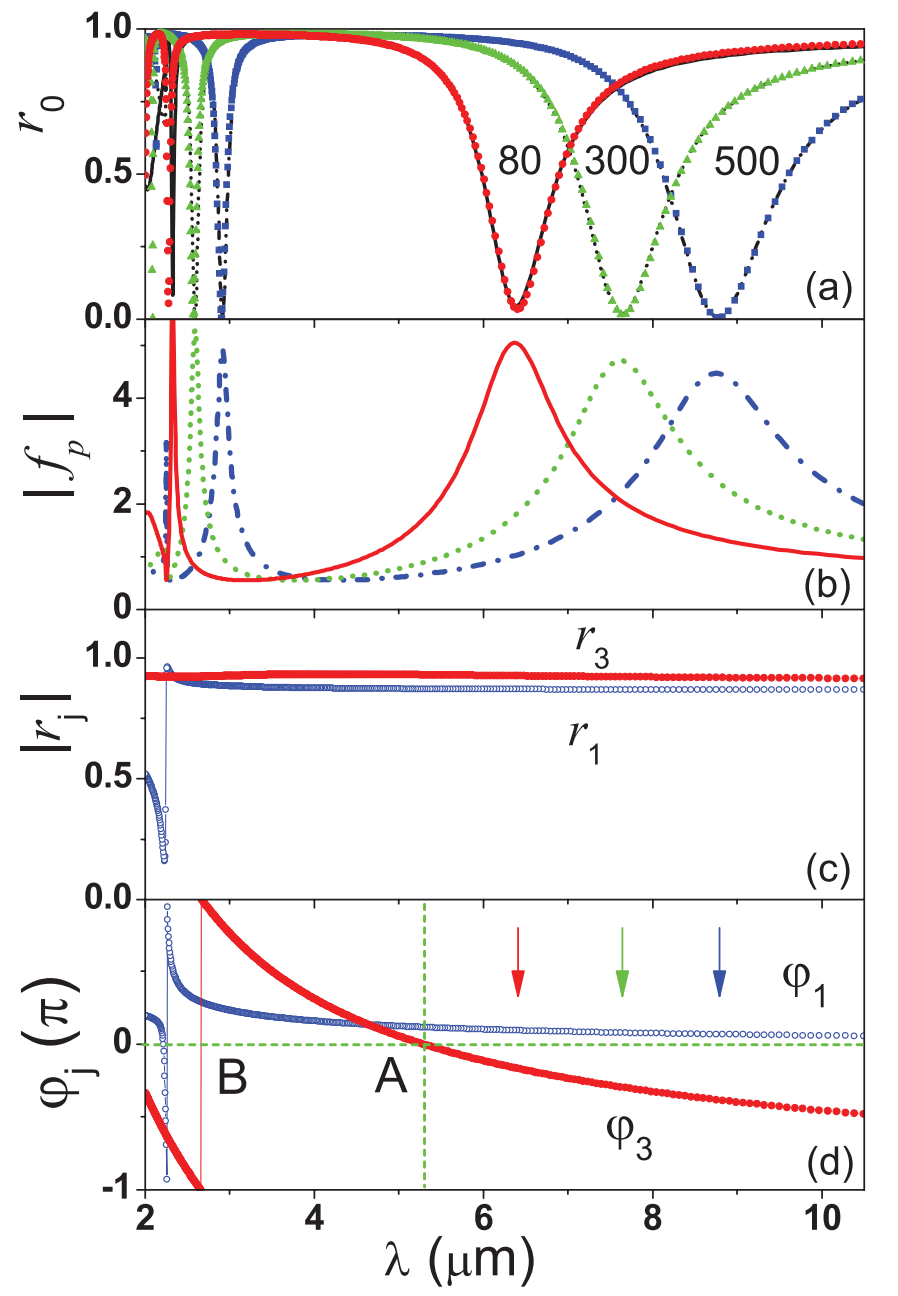

FIG. 2. (Color online) (a) Numerically simulated (symbols) and analytically calculated (lines) reflection spectra; (b) $\left|f_{p}\right|$ as a function of wavelength; (c) amplitude; and (d) phase shift of $r_{j}$ at the slit openings. The arrows indicate the vertical FP-like resonances. Here, $t=20 \mathrm{~nm}$ and $h=80,300$, and $500 \mathrm{~nm}$.
We developed an analytical model, which is crucial for an intuitive understanding of the aforementioned effect. We expanded the fields on the incident side and in the spacer into a Fourier series and treated the horizontal metal surfaces using the surface-impedance boundary conditions. For the narrow slits that satisfy the conditions $a \ll \lambda$ and $h \geqslant a$, we only considered the fundamental slit mode, which has an effective propagation constant $q_{0}=k_{0} n_{h}$, where $k_{0}=\omega / c$ and $n_{h}=$ $\sqrt{1+i \lambda / \pi a \varepsilon_{m}^{1 / 2}}$ is the effective index of slits. ${ }^{26}$ We derived an analytic solution for the zero-order reflection as follows:

$$
r_{0}=\left|\kappa-\frac{\eta\left(1+r_{3} e^{2 i q_{0} h}\right)}{\left(1+\theta_{1}\right)\left(1-r_{1} r_{3} e^{2 i q_{0} h}\right)}\right|^{2} .
$$

Here, $r_{j}=\left(1-\theta_{j}\right) /\left(1+\theta_{j}\right)$ is the equivalent reflection coefficient of the slit mode at the upper $(j=1)$ or lower $(j=3)$ slit openings, where $\theta_{1}=\alpha \sum_{n} g_{n}^{2} /\left(1+\sigma_{n}\right), \theta_{3}=$ $\alpha \sum_{n} \mu_{n} g_{n}^{2} /\left(1+\tau_{n}\right)$, and the remaining quantities are defined in Ref. 27. Note that the summation in $\theta_{j}$ involves all the diffraction orders above or below the grating, implying that the boundary conditions at the slit openings are dressed by the diffracted surface waves of the periodic structure. Equation (1) can give rather precise results, as demonstrated below, and it reveals the essence of the physics. For example, the reflection of light is related to an FP-like resonance factor $f_{p}=\left(1-r_{1} r_{3} e^{2 i q_{0} h}\right)^{-1}$, suggesting that the metal slits may act as resonant cavities under certain conditions.

The reflection spectra calculated using Eq. (1) are shown in Fig. 2(a) by the lines, which track the symbols representing the numerical results closely. This suggests that our analytic expression captures the underlying physics of system very well. To clarify the origins of reflection dips, the absolute value of FP resonance factor $\left|f_{p}\right|$ as a function of wavelength is plotted in Fig. 2(b). One can see that there is a oneto-one correspondence between each reflection dip and the maximum of $\left|f_{p}\right|$. Thus, the long wavelength reflection dips can be attributed to the FP-like resonance in the metallic slits. The narrow dips at short wavelength are related to surface resonances, ${ }^{20,28,29}$ which cause phase jumps in a narrow frequency range [see Fig. 2(d)]. The rapid change of phase allows for the possibility of satisfying the FP resonance condition, but the peak is very narrow; the FP resonance in turn feeds energy into the surface mode. Such effect would facilitate EOT phenomena if the system allows for transmission. ${ }^{20,28}$

Given that the length of FP cavities is very small compared with the wavelength, the effect may appear surprising. Here, by expressing the equivalent reflection coefficient $r_{j}$ of the slit mode as $r_{j}=\left|r_{j}\right| e^{i \varphi_{j}}$, the FP-like resonance condition becomes

$$
\varphi_{1}+\varphi_{3}+\frac{4 \pi n_{h}}{\lambda} h=2 \pi m
$$

where $\left|r_{j}\right|$ and $\varphi_{j}$ are the amplitude and phase shift of $r_{j}$ at the slit openings. Using the analytic expressions, we find that $\left|r_{1}\right|$ and $\left|r_{3}\right|$ depend weakly on the wavelength and both are close to unity in the long wavelength region [see Fig. 2(c); the rapid change of $\left|r_{1}\right|$ at $2250 \mathrm{~nm}$ corresponds to the surfaceplasmon polariton (SPP) mode on the incident side]. Thus the slit openings are strong reflecting boundaries and the cavity mode can be well confined. 
In our structure the diffracted surface waves above and below the grating are coupled with the slit mode to induce an FP-like resonance, as described by Eq. (2), where the role of surface waves is manifested in the phase shift. The dependence of the phase shift on the wavelength is shown in Fig. 2(d). The phase shift at the top of the slit, $\varphi_{1}$, is a phase jump at $2250 \mathrm{~nm}$ followed by a gradual decrease from $\pi$ to zero. In contrast, the phase shift at the bottom, $\varphi_{3}$, is a phase jump near point B $(2660 \mathrm{~nm})$ followed by a decrease to negative values by crossing a zero-phase point A $(5280 \mathrm{~nm})$. Here, the variation of $\varphi_{3}$ is linked to the narrow air spacer sandwiched between the two metal layers, where two types of resonance modes are involved. One resonance mode corresponds to the poles of $\mu_{n}$ and $\theta_{3}$, satisfying the equation $k_{0} \sqrt{\varepsilon_{d}} \sin \varphi+2 \pi m / d=$ $\pm k_{0} n_{t}\left(n_{t}=\sqrt{1+2 \delta / t}\right.$ is the effective index of the gold-air -gold region ${ }^{26}$ ). This is the extended SPP mode on the planar metal film, responsible for the phase jump at point $\mathrm{B}$. The zero-phase point $\mathrm{A}$ corresponds to a near-zero $\theta_{3}\left[\operatorname{Im}\left(\theta_{3}\right)=0\right]$, and the spectral positions can be approximated by $2 n_{t}(d-$ $a)=m \lambda$. This is just the horizontal FP-like resonance beneath the metal stripes (the resonance has a strong magnetic character and is basically the same type of resonance that gives rise to a high surface impedance ${ }^{30}$ ).

We found that for a larger slit width, the coupling between adjacent metal stripes is weak, and the localized (horizontal) mode causes a steep phase variation near point A. For the narrow slits of which we are most interested, the horizontal mode will become delocalized due to the interstripe coupling, and the phase variation near point A becomes extremely gentle. This unique phase shift together with an unusual value of $\varphi_{1}$ may produce a new and deep subwavelength FP-like resonance. On the right-hand side of point A (far from the horizontal resonances), $\varphi_{1}$ and $\varphi_{3}$ have opposite signs, and the sum of them is negative but small. Thus a small slit length may ensure a zero-order FP-like resonance. For example, at $\lambda=6400 \mathrm{~nm}$, we have $\varphi_{1}=0.095 \pi$ and $\varphi_{3}=-0.164 \pi$. According to Eq. (2), a slit length of $80 \mathrm{~nm}$ (the slit width is $50 \mathrm{~nm}$ ), which is two orders of magnitude smaller than the wavelength, is sufficient for forming an FP-like resonance.

Besides the strong dependence of resonance on the subwavelength cavity length, the effect can further be manipulated by using the phase shift at the slit openings [see Figs. 3(a) and 3(b)]. Figure 3(b) presents the phase curves for two spacer thicknesses $t=20 \mathrm{~nm}$ and $t=10 \mathrm{~nm}(h=80 \mathrm{~nm})$. With the decrease of $t$ (especially when $t<30 \mathrm{~nm}$ ), the zero-phase point $\mathrm{A}$ (determined by $n_{t}$ ) and the associated vertical FP-like resonance (denoted by the arrow) will shift to the longer wavelength. This means we can obtain very deep subwavelength FP-like resonances in the slits by squeezing the spacer thickness. This trend is seen clearly in the reflection spectrum, as shown in Fig. 3(a), where the dark or redcolored part highlights the FP-induced reflection minimum. Interestingly, when $t$ is down to $20 \mathrm{~nm}$, a further change of $t$ by only $1 \mathrm{~nm}$ can lead to a 60-nm wavelength shift of the FP-like resonance. If an infrared Fourier spectroscopy with a resolution of $2 \mathrm{~cm}^{-1}(\sim 8.2 \mathrm{~nm}$ at $6400 \mathrm{~nm})$ is used, the accuracy of displacement measurement can reach $0.14 \mathrm{~nm}$. This enables the detection of tiny motion or oscillation with ultrahigh sensitivity. In addition, Fig. 4 presents the electricfield distribution for two spacer thicknesses and resonance
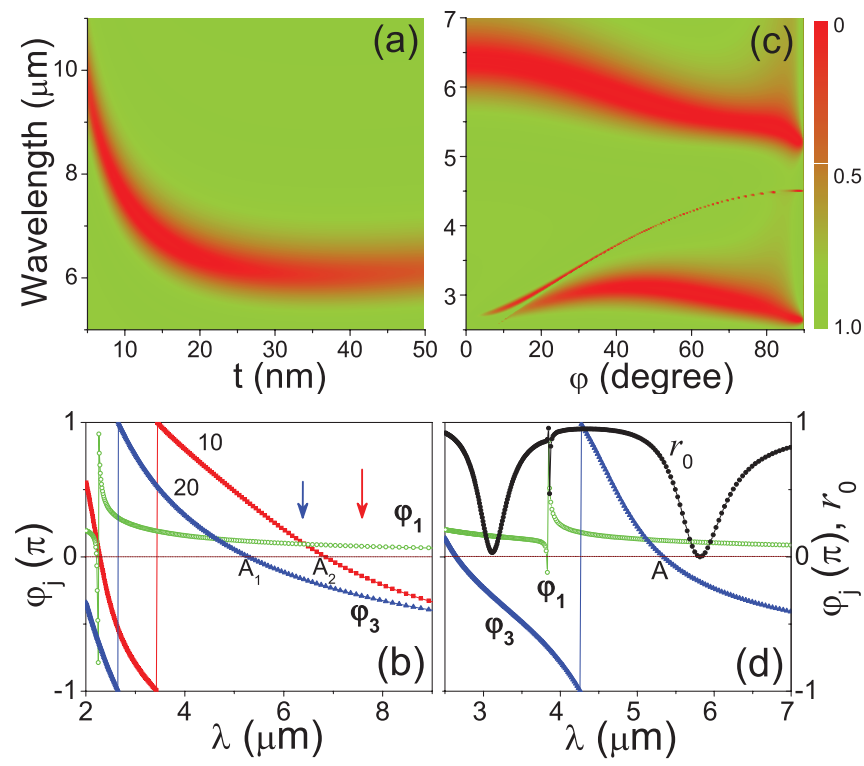

FIG. 3. (Color online) (a) Reflection spectrum as a function of wavelength and spacer thickness, and (b) phase shift $\varphi_{j}$ of the slit mode for $t=20$ and $10 \mathrm{~nm}\left(\varphi=0^{\circ}\right)$. (c) Reflection spectrum as a function of wavelength and incident angle $(t=20 \mathrm{~nm})$, and (d) phase shift and reflection for $\varphi=45^{\circ}$. Here, we fix $h=80 \mathrm{~nm}$.

wavelengths (here $h=80 \mathrm{~nm}$; the results for $h=300 \mathrm{~nm}$ and $500 \mathrm{~nm}$ are very similar). One can see that in both cases the deep subwavelength FP-like resonance is strongly excited, and a significant portion of energy can be stored in the FP cavity.

Figure 3(c) shows the calculated reflection spectrum as a function of wavelength and incident angle $\varphi$, where the dominant FP-like resonance blueshifts as $\varphi$ increases $(h=$ $80 \mathrm{~nm}$ and $t=20 \mathrm{~nm}$ ). To understand this effect, Fig. 3(d) presents the phase curves and reflection spectrum at $\varphi=45^{\circ}$. Compared with Fig. 3(b) where $\varphi=0^{\circ}$, the sharp-phase jumps
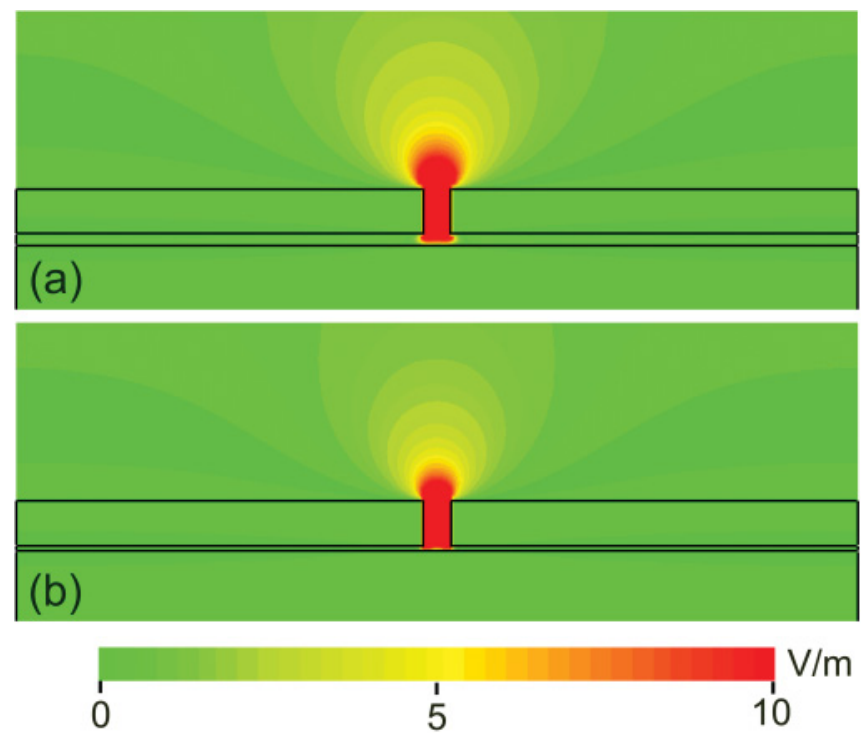

FIG. 4. (Color online) Electric-field patterns ( $E x)$ for two spacer thicknesses and vertical FP-like resonance wavelengths: (a) $t=20 \mathrm{~nm}$ and $\lambda=6400 \mathrm{~nm}$; (b) $t=10 \mathrm{~nm}$ and $\lambda=7570 \mathrm{~nm}$. Here, $h=80 \mathrm{~nm}$, $\varphi=0^{\circ}$, and the incident field is set at $1 \mathrm{~V} / \mathrm{m}$ in the simulation. 

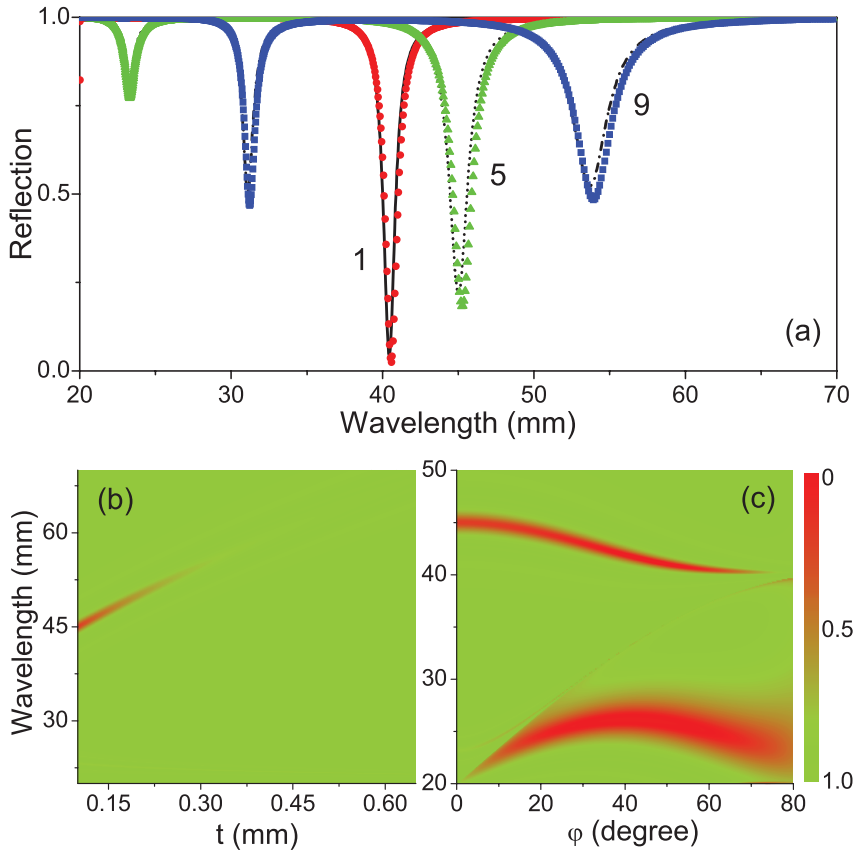

FIG. 5. (Color online) (a) Numerically simulated (symbols) and analytically calculated (lines) reflection spectra at microwave frequencies $(t=0.1 \mathrm{~mm}$ and $h=1,5$, and $9 \mathrm{~mm})$. The reflection as a function of wavelength and spacer thickness or incident angle is shown in (b) $\left(h=5 \mathrm{~mm}, \varphi=0^{\circ}\right)$ and (c) $(h=5 \mathrm{~mm}, t=0.1 \mathrm{~mm})$, respectively. Here, $d=20 \mathrm{~mm}, a=1 \mathrm{~mm}$, and $\varepsilon_{d}=1$.

in Fig. 3(d) corresponding to the extended surface modes are redshifted, but the zero-phase point A remains almost unchanged [see the green (gray) circles and blue (medium gray) triangles]. As a consequence, the phase variation near point A becomes drastic, and the phase-matching condition Eq. (2) for the vertical FP-like resonance is satisfied at a reduced wavelength. Despite not being our focus, we will point out that a deep subwavelength (zero-order) FP-like resonance also appears near $3000 \mathrm{~nm}$ for the non-normal incidence. Here, the zero-phase point near $2650 \mathrm{~nm}$ corresponds to the second-order resonance in the spacer (which is inactive at normal incidence). Additionally, the surface mode on the incident side is accompanied by a narrow reflection dip located at $3840 \mathrm{~nm}$ in Fig. 3(d). This dip may mix with the resonance mode near $3000 \mathrm{~nm}$ if we vary the incident angle, giving rise to an anticrossinglike coupling behavior in Fig. 3(c).

The effect may be extended from infrared to low-frequency band. As an example, Fig. 5 presents the theoretical results for microwave frequencies. In this regime the medium on the incident side was set as air with $\varepsilon_{d}=1$, and the gold was modeled with $\varepsilon_{m}=1+i \sigma / \varepsilon_{0} \omega$, where $\sigma=4.1 \times 10^{7} \mathrm{~S} / \mathrm{m}$. Figure 5(a) shows the numerically simulated (symbols) and analytically calculated (lines) reflection spectra for different cavity lengths. Again, the analytical and numerical results agree with each other. For $h=1 \mathrm{~mm}$, the vertical (zero-order) FP-like resonance locates at a wavelength of $41 \mathrm{~mm}$, which is much larger than the FP cavity length. Moreover, in accordance with Eq. (2), the resonance wavelength exhibits a significant redshift as $h$ increases (the dips at $23 \mathrm{~mm}$ and $31 \mathrm{~mm}$ originate from the first-order FP-like resonance of slits). Figure 5(b) presents the reflection spectrum as a function of wavelength and spacer thickness $(h=5 \mathrm{~mm})$. Different from the result in the infrared band [Fig. 3(a)], here, as $t$ increases, the dominant FP-like resonance redshifts instead. In the microwave regime, the position of zero-phase point $\mathrm{A}$ is almost independent of $t\left(n_{t} \approx 1\right)$. However, the larger the spacer thickness, the more delocalized the horizontal FP mode. Thus, the phase variation near point $\mathrm{A}$ becomes gentler, resulting in an even larger resonance wavelength. In addition, the dependence of reflection spectrum on the incident angle is also shown in Fig. 5(c), and the behavior is similar to that in the infrared band, as shown in Fig. 3(c). These results point to the possibility of manipulating deep subwavelength FP-like resonances in microwave frequencies.

\section{CONCLUSIONS}

In summary, the optical properties of a sandwiched reflection grating have been studied. Our results show that the open-ended metallic slits may act as deep subwavelength FP cavities, which can operate with the resonance wavelength two orders of magnitude larger than the cavity size. The effect may be used to realize the dynamically modulated light reflections, measure the displacement of objects with subnanometer accuracy, and drive the mechanical motions with the enhanced fields.

\section{ACKNOWLEDGMENTS}

This work was supported by the Hong Kong Research Grants Council (Grant No. 600209), the National Basic Research Program of China (Grant No. 2012CB921502), the HKUST internal Grant No. SRFI1SC07, and the National Natural Science Foundation of China (Grant No. 11174146). Computation resources were provided by the Hong Kong Shun Hing Education and Charity Fund.

\footnotetext{
*Corresponding author: cphuang@ njut.edu.cn

†phchan@ust.hk

${ }^{1}$ C. F. Bohren and D. R. Huffman, Absorption and Scattering of Light by Small Particles (John Wiley, New York, 1983).

${ }^{2}$ J. A. Schuller, E. S. Barnard, W. Cai, Y. C. Jun, J. S. White, and M. I. Brongersma, Nat. Mater. 9, 193 (2010).

${ }^{3}$ V. M. Shalaev, Nat. Photon. 1, 41 (2007).

${ }^{4}$ S. Linden, C. Enkrich, M. Wegener, J. Zhou, T. Koschny, and C. M. Soukoulis, Science 306, 1351 (2004).
}

${ }^{5}$ J. Valentine, S. Zhang, T. Zentgraf, E. Ulin-Avila, D. A. Genov, G. Bartal, and X. Zhang, Nature 455, 376 (2008).

${ }^{6}$ X. Zhang and Z. W. Liu, Nat. Mater. 7, 435 (2008).

${ }^{7}$ M. Silveirinha and N. Engheta, Phys. Rev. B 75, 075119 (2007).

${ }^{8}$ M. Choi, S. H. Lee, Y. Kim, S. B. Kang, J. Shin, M. H. Kwak, K. Y. Kang, Y. H. Lee, N. Park, and B. Min, Nature 470, 369 (2011).

${ }^{9}$ J. B. Pendry, D. Schurig, and D. R. Smith, Science 312, 1780 (2006).

${ }^{10}$ H. Chen, C. T. Chan, and P. Sheng, Nat. Mater. 9, 387 (2010).

${ }^{11} \mathrm{G}$. Von Trentini, IEEE Trans. Antennas Propag. 4, 666 (1956). 
${ }^{12}$ L. Zhou, H. Q. Li, Y. Qin, Z. Y. Wei, and C. T. Chan, Appl. Phys. Lett. 86, 101101 (2005).

${ }^{13}$ H. Q. Li, J. M. Hao, L. Zhou, Z. Y. Wei, L. K. Gong, H. Chen, and C. T. Chan, Appl. Phys. Lett. 89, 104101 (2006).

${ }^{14}$ A. Ourir, A. de Lustrac, and J. Lourtioz, Appl. Phys. Lett. 88, 084103 (2006).

${ }^{15}$ J. Hao, L. Zhou, and M. Qiu, Phys. Rev. B 83, 165107 (2011).

${ }^{16}$ H. T. Chen, Opt. Express 20, 7165 (2012).

${ }^{17}$ S. Thongrattanasiri, F. H. L. Koppens, and F. J. Garcia de Abajo, Phys. Rev. Lett. 108, 047401 (2012).

${ }^{18}$ Y. Takakura, Phys. Rev. Lett. 86, 5601 (2001).

${ }^{19}$ A. P. Hibbins, M. J. Lockyear, and J. R. Sambles, J. Appl. Phys. 99, 124903 (2006).

${ }^{20}$ J. A. Porto, F. J. Garcia-Vidal, and J. B. Pendry, Phys. Rev. Lett. 83, 2845 (1999).

${ }^{21}$ M. H. Lu, X. K. Liu, L. Feng, J. Li, C. P. Huang, Y. F. Chen, Y. Y. Zhu, S. N. Zhu, and N. B. Ming, Phys. Rev. Lett. 99, 174301 (2007).

${ }^{22}$ A. P. Hibbins, J. R. Sambles, C. R. Lawrence, and J. R. Brown, Phys. Rev. Lett. 92, 143904 (2004).
${ }^{23}$ Y. Todorov, A. M. Andrews, I. Sagnes, R. Colombelli, P. Klang, G. Strasser, and C. Sirtori, Phys. Rev. Lett. 102, 186402 (2009).

${ }^{24}$ Z. Y. Wei, H. Q. Li, Y. Cao, C. Wu, J. Z. Ren, Z. H. Hang, H. Chen, D. Z. Zhang, and C. T. Chan, New J. Phys. 12, 093020 (2010).

${ }^{25}$ P. Jouy, Y. Todorov, A. Vasanelli, R. Colombelli, I. Sagnes, and C. Sirtori, Appl. Phys. Lett. 98, 021105 (2011).

${ }^{26}$ This index is increased due to the coupling between the photons and surface charges on the metal walls. In near- and mid-infrared frequency range, $n_{h} \approx \sqrt{1+2 \delta / a}$, where $\delta=22 \mathrm{~nm}$ is the skin depth of gold; see S. Collin, F. Pardo, and J. Pelouard, Opt. Express 15, 4310 (2007).

${ }^{27}$ Here $\quad \mu_{n}=\left(1-\rho_{n} e^{2 i k_{0} v_{n} t}\right) /\left(1-\rho_{n}^{2} e^{2 i k_{0} v_{n} t}\right), \quad \rho_{n}=\left(1-\tau_{n}\right) /(1+$ $\left.\tau_{n}\right), \quad$ and $\quad g_{n}=\sin c\left(k_{0} \gamma_{n} a / 2\right) ; \quad \gamma_{n}=\sqrt{\varepsilon_{d}} \sin \varphi+n \lambda / d, \quad u_{n}=$ $\sqrt{\varepsilon_{d}-\gamma_{n}^{2}}, v_{n}=\sqrt{1-\gamma_{n}^{2}}, \sigma_{n}=u_{n} \sqrt{\varepsilon_{m}} / \varepsilon_{d}$, and $\tau_{n}=v_{n} \sqrt{\varepsilon_{m}} ; \kappa=$ $\left(\sigma_{0}-1\right) /\left(\sigma_{0}+1\right), \eta=2 g_{0}^{2} \alpha \sigma_{0} /\left(1+\sigma_{0}\right)^{2}$, and $\alpha=n_{h} a \sqrt{\varepsilon_{m}} / d$.

${ }^{28}$ T. W. Ebbesen, H. J. Lezec, H. F. Ghaemi, T. Thio, and P. A. Wolff, Nature 391, 667 (1998).

${ }^{29}$ B. Auguie and W. L. Barnes, Phys. Rev. Lett. 101, 143902 (2008).

${ }^{30}$ D. Sievenpiper, L. Zhang, R. F. Jimenez Broas, N. G. Alexopolous, and E. Yablonovitch, IEEE Trans. Microwave Theory Tech. 47, 2059 (1999). 